\title{
Akute febrile neutrophile Dermatose (Sweet-Syndrom) - Eine seltene Komplikation der Colitis ulcerosa
}

\section{A. De Donno \\ P. von den Driesch}

\section{Acute Febrile Neutrophilic Dermatosis (Sweet's Syndrome) - A Rare Complication of Ulcerative Colitis}

\section{Zusammenfassung}

Die akute febrile neutrophile Dermatose (Sweet Syndrom) wurde erstmals von Robert Douglas Sweet 1964 als akutes Krankheitsbild unbekannter Ätiologie beschrieben. Klinisch ist das Sweet-Syndrom durch plötzliches Auftreten von schmerzhaften sukkulenten Erythemen mit Fieber und schlechtem Allgemeinzustand gekennzeichnet und zeigt meist ein gutes Ansprechen auf systemische Steroide. Es wird, wie beispielsweise auch das Pyoderma gangraenosum zur Gruppe der reaktiven neutrophilen Dermatosen gezählt und als Hypersensitivitätsreaktion bei systemischen Erkrankungen, häufig nach Infekten, bei Kollagenosen oder Störungen der Hämatopoese, interpretiert. In seltenen Fällen tritt das Sweet-Syndrom in Zusammenhang mit entzündlichen Darmerkrankungen auf. Wir zeigen einen Fall, bei dem ein Sweet-Syndrom im Zusammenhang mit einer Colitis ulcerosa auftrat.

\section{Abstract}

The acute febrile neutrophilic dermatosis (Sweet's syndrome) was first described in 1964 by Robert Douglas Sweet as an acute disease of unknown etiology. The characteristic clinical features are the sudden onset of painful erythematous plaques with fever and malaise. In most cases, a good response to systemic corticosteroid is characteristic. Sweet's syndrome may be considered as part of a continuous spectrum of reactive neutrophilic dermatoses such as pyoderma gangrenosum and it has been considered as a hypersensitivity reaction to systemic disorders, mostly infections, autoimmune diseases, or hematopoetic diseases. In some cases, Sweet's syndrome appears in connection with inflammatory bowel diseases. We present a case of Sweet's syndrome in association with ulcerative colitis.

\section{Einleitung}

Die akute febrile neutrophile Dermatose (Sweet-Syndrom), ist eine akut verlaufende fieberhafte Erkrankung, die mit einer neutrophilen Leukozytose und schmerzhaften, sukkulenten Erythemen vor allem im Gesicht, am Nacken und an den Extremitätenstreckseiten einhergeht $[1,2]$. Sie tritt bevorzugt bei Frauen zwischen dem dreißigsten und fünfzigsten Lebensjahr auf [2]. Unterschieden werden vier Gruppen des Sweet-Syndroms, das klas- sische oder idiopathische, das paraentzündliche, das häufig nach Infekten der oberen Luftwege, bei Kollagenosen und selten auch bei entzündlichen Darmerkrankungen auftritt, das paraneoplastische, meist in Zusammenhang mit hämatologischen Erkrankungen [2] und eine allerdings sehr seltene medikamenteninduzierte Variante [2].

Institutsangaben

Klinik für Dermatologie und Allergologie, Zentrum für Hautkrankheiten, Klinikum Stuttgart

(Direktor: Prof. Dr. P. von den Driesch)

Widmung

Herrn Prof. Dr. O. P. Hornstein zum 80. Geburtstag gewidmet

Korrespondenzadresse

Prof. Dr. Peter von den Driesch · Klinik für Dermatologie und Allergologie Klinikum Stuttgart · Prießnitzweg 24

70374 Stuttgart · E-mail: pdriesch@kbc-intern.de

Bibliografie

Akt Dermatol 2006; 32: 368-370 @ Georg Thieme Verlag KG Stuttgart · New York

DOI 10.1055/s-2006-925434 · ISSN 0340-2541 


\section{Anamnese}

Der 39-jährige Patient stellt sich mit seit zwei Tagen bestehenden, sich vom Nacken auf die Extremitäten ausbreitenden, brennenden Erythemen vor. Er beklagt zudem ein allgemeines Krankheitsgefühl mit hohem Fieber über $39^{\circ} \mathrm{C}$, Kopf- und Gliederschmerzen.

Wenige Wochen zuvor sei nach einer Darmspiegelung die Diagnose einer Colitis ulcerosa vom niedergelassenen Gastroenterologen gestellt worden, weswegen der Patient bis vor zwei Tagen Salofalk (Mesalazin) Tabletten eingenommen habe. Diese habe er seit Auftreten der Hautveränderungen weggelassen, da er einen Zusammenhang zwischen der Medikamenteneinnahme und dem Auftreten des Ausschlags befürchte.

\section{Dermatologischer und körperlicher Aufnahmebefund}

(Abb. 1 a u. b)

Im Gesicht, am Hals sowie an den Extremitätenstreckseiten bis zu $4 \times 4 \mathrm{~cm}$ große, einzelstehende, scharf begrenzte, sukkulente, polsterförmig erhabene Erytheme.

\section{Labor}

Pathologisch verändert: Leukozyten $14300 / \mu l$, Neutrophile $82 \%$, CRP 23,5 mg/dl, Hb 12,8 g/dl, Gamma-GT 59 U/l.

Normwerte für restliches Blutbild, Leber-, und Nierenwerte, Elektrolyte.

\section{Histologie (Abb. 2)}

Fokale Parakeratose, vereinzelt nekrotische Keratinozyten. Massives subepidermales Ödem. In der retikulären Dermis dichtes, bandförmiges, entzündliches Infiltrat aus überwiegend neutrophilen Granulozyten mit Leukozytoklasie, wenigen Lymphozyten und Histiozyten bei geringen Vaskulitis-Zeichen. Beurteilung: Sehr gut zu Sweet-Syndrom passend.

\section{Therapie und Verlauf}

Es kam zu einer raschen Abheilung unter Methylprednisolon per os in ausschleichender Dosierung mit initial $80 \mathrm{mg}$ täglich in Kombination mit Indometacin $50 \mathrm{mg}$ 3-mal täglich. Lokal applizierten wir Klasse-III-Steroide mit antiseptischem Zusatz.

Zur Behandlung der Colitis ulcerosa wurde zu einer Wiederaufnahme der Mesalazin-Therapie geraten. Diese wurde komplikationslos vertragen.

\section{Diskussion}

Die akute febrile neutrophile Dermatose, nach ihrem Erstbeschreiber auch als Sweet-Syndrom bekannt, zeichnet sich durch plötzliches Auftreten von schmerzhaften sukkulenten Erythemen mit Fieber und reduziertem Allgemeinbefinden aus [2]. Die Ätiologie und Pathogenese sind unbekannt [1]. In der Literatur sind nur 30 Fälle beschrieben, bei denen das Sweet-Syndrom mit chronisch entzündlichen Darmerkrankungen assoziiert ist
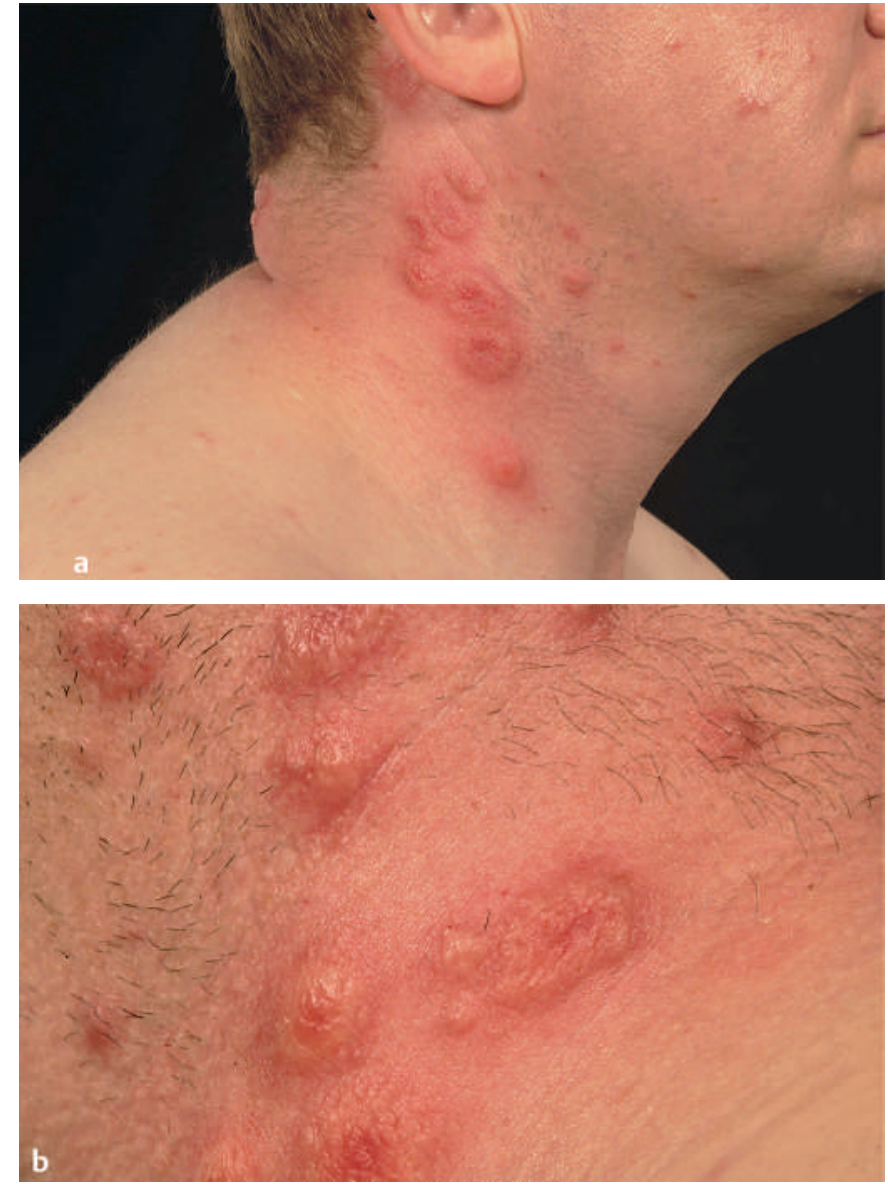

Abb. 1 a,b Die Bilder zeigen die typischen sukkulenten indurierten Plaques des Sweet-Syndroms. In der näheren Aufnahme sehr schön der Aspekt des einzeln stehenden erhabenen Herdes („,relief of a rocky island“).

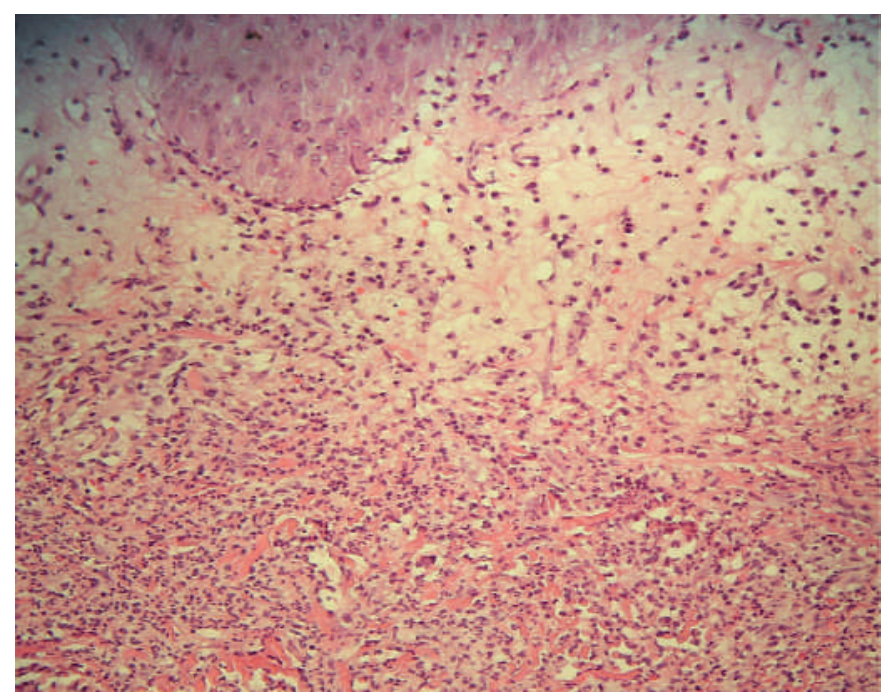

Abb. 2 Histologie des Sweet-Syndroms: Eher einzelne epidermotrope Neutrophile, das klassische prominente subepidermale Ödemband sowie darunter das bandförmige Infiltrat, neutrophilenreich, mit geringer bis fehlender Vaskulitis. 
$[1,3]$. Es wird, wie auch das Pyoderma gangraenosum, das Erythema elevatum diutinum, die subcorneale Pustulose und andere zur Gruppe der reaktiven neutrophilen Dermatosen gezählt $[2,5]$. Die Erkrankungen dieser Gruppe haben histologisch dichte Infiltrate aus neutrophilen Granulozyten mit variablem Anteil vaskulitischer Elemente gemeinsam [2,4,5]. Die klinisch oft deutlich zu unterscheidenden Erkrankungen können histologisch fließende Übergänge zeigen [2,5]. Als in der Literatur am häufigsten beschriebene extraintestinale Manifestation bei entzündlichen Darmerkrankungen wie Morbus Crohn und Colitis ulcerosa ist aus dieser Gruppe das Pyoderma gangraenosum zu nennen [5].

Sowohl bei den in der Literatur beschriebenen Fällen [1-3], als auch bei unserem Patienten ist ein deutlicher zeitlicher Zusammenhang zwischen dem Auftreten der Hautveränderungen des Sweet-Syndroms und der Erstdiagnose oder einem aktiven Schub einer entzündlichen Darmerkrankung wie der Colitis ulcerosa zu erkennen $[1-4]$. Allerdings sind auch Fälle beschrieben, bei denen die Darmerkrankung keine oder kaum Aktivität zeigte und es dennoch zum Auftreten einer reaktiven neutrophilen Dermatose kam [5]. Daher sollten die reaktiven neutrophilen Dermatosen als eigenständige Erkrankungen angesehen werden. Interessant ist die Rolle des Zytokins TNF- $\alpha$, das sowohl bei den „Grunderkrankungen“ als auch bei den neutrophilen Dermatosen vermutlich eine sehr wichtige Rolle spielt [6].

\section{Literatur}

${ }^{1}$ Schlegel Gomez R, Kiesewetter F, von den Driesch P, Hornstein OP. Sweet-Syndrom und Erythema nodosum bei Morbus Crohn. Hautarzt 1990; 41: 390-401

2 von den Driesch P. Sweet's syndrome. J Am Acad Dermatol 1994; 31 : 535-556

${ }^{3}$ Ytting H, Band D, Munkholm P. Sweet's syndrome - an extraintestinal manifestation in inflammatory bowel disease. Digestion 2005; 72 : 195-200

${ }^{4}$ Paoluzi OA, Crispino P, Amantea A et al. Diffuse febrile dermatosis in a patient with active ulcerative colitis under treatment with steroids and azathioprine: a case of Sweet's syndrome Case report and review of literature. Dig Liver Dis 2004; 36: 361 - 366

${ }^{5}$ von den Driesch P. Pyoderma gangrenosum: a report of 44 cases with follow up. Br J Dermatol 1997; 137: 1000 - 1005

${ }^{6}$ von den Driesch P, Simon MJR, Djawari D, Wassmuth R. Analysis of HLA antigens in Caucasian patients with acute febrile neutrophilic dermatosis (Sweet's syndrome). J Am Acad Dermatol 1997; 37: 276 277

\section{Buchbesprechung}

\section{Asthma und allergische Rhinitis - eine Erkrankung mit zwei Gesichtern}

C. Bachert, B. Lange, J. C. Virchow

Stuttgart: Thieme, 2005. 88 S., 16 Abb., 17 Tab., Kart. 14,95€,

ISBN 3-13-133991-8

Die Autoren Bachert aus Gent, Virchow aus Rostock und Lange aus Düsseldorf sind die Herausgeber eines Fachbuches, das sich mit Asthma und allergischer Rhinitis befasst. In der Kürze, die 88 Seiten und das Taschenbuchformat gebieten, werden im ersten kurzen Kapitel die immunologischen Parallelen der beiden Krankheitsbilder erläutert; es folgen Kapitel zur Epidemiologie, zur Pathophysiologie, zur Diagnostik und zu aktuellen Leitlinien der Therapie. Im Zentrum dieser beiden umfangreicheren Kapitel zu Diagnostik und Therapie stehen neue Klassifikationen, Schweregraddefinitionen, Differenzialdiagnosen, Bewertungs-kriterien der Diagnostik, Ampelschemata, Stufenpläne für die Therapie und Erläuterungen zu den internationalen Leitlinien „Allergic Rhinitis and its Impact on Asthma (ARIA)“, die von einer WHO unterstützten Arbeitsgruppe erstellt wurden. Berücksichtigt wird zusätzlich auch die Asthmaleitlinie der „Global Initiative for Asthma (GINA)“. Dabei geht es in den praxisnahen Ausführungen dieses Bandes vor allem um die Patienten - Erwachsene und Kinder - die unter Asthma und Rhinitis leiden und das sind je nach Studie bis zu $40 \%$, allein bei den Erwachsenen mit allergischer Rhinitis, und 30-70\%, bei denen mit Asthma bronchiale, mit jeweils höheren Prävalenzen bei Kindern. Der Schreibstil ist verständlich bei hohem wissenschaftlichen Niveau. Die Verständlichkeit wird durch hervorragende Abbildungen und Tabellen unterstützt, ergänzt durch eine „Checkliste“ der wichtigsten Punkte am Ende jedes Kapitels. Aktualität besitzt der Band in hohem Maße und ist eigentlich eine Pflichtlektüre für Allergologen aller Fachrichtungen - um auf dem neuesten Stand zu sein. Das Büchlein ist aber aufgrund der guten Didaktik und des komprimierten Inhalts auch Studenten zu empfehlen.

Christiane Bayerl, Wiesbaden 